\title{
LA FORMACIÓN DE INSTRUCTORES EN TIC USANDO AMBIENTES VIRTUALES DE APRENDIZAJE
}

\section{TRAINING INSTRUCTORS ON I.T. BY MEANS OF THE USE OF VIRTUAL LEARNING ENVIRONMENTS (SENA NATIONWIDE PILOT TEST)}

\author{
(Prueba Piloto Nacional SENA) \\ Adriana Lizeth Buitrago \\ abuitrago@sena.edu.co
}

\section{ABSTRACT}

Developing the SENA1 Virtual Educative Community project a process began in the year 2001 to establish the linings that would allow the concepts about formation in virtual environments to be standardized, such process counted with the participation of civil servants from different regions of the country who have managed the "New Information and Communication Technologies applied to Formation" course, and ended in the implementation of a national pilot test of that course, using the existing CD-ROM multimedia as content guide, and the internet as interaction and collaboration environment. The results of this pilot test will be used as a prime matter to begin the formation of alumnae through this strategy.

Keywords: Virtual Environments for Learning, Self training, Telematic Networks, IT Information and Communication Technology

\section{Resumen}

El desarrollo del Proyecto de Comunidad Educativa Virtual SENA ${ }^{1}$ se inició en el año 2001 un proceso para establecer los lineamientos que permitieran estandarizar los conceptos sobre Formación en Ambientes Virtuales de Aprendizaje, con la participación de funcionarios de diferentes regiones del país que han tutorizado el curso de Nuevas Tecnologías de la Información y la Comunicación Aplicadas a la Formación, proceso que culminó con el desarrollo de una prueba piloto nacional de dicho curso, usando como guía de contenidos los CD Roms multimediales existentes y como ambiente de interacción y colaboración Internet. Los resultados de esta prueba piloto serán usados como insumo principal para iniciar la formación de alumnos a través de esta estrategia.

Palabras Clave: ambientes virtuales de aprendizaje, autoformación, redes telemáticas, TIC - Tecnologías de Información y Comunicación.

\footnotetext{
${ }^{1}$ Servicio Nacional de Aprendizaje - SENA. Institución de Formación Profesional Integral, adscrito al Ministerio de Trabajo y Seguridad Social de Colombia.
} 


\section{AnTECEDENTES}

En el año 1999 se finalizó el trabajo de codesarrollo con la firma Ihardun Multimedia Coop. de España, de un curso multimedial sobre Nuevas Tecnologías de la Información y la Comunicación Aplicadas a la Formación, para ser implementado dentro del plan de formación y mejoramiento continuo de los instructores del SENA en todo el país ${ }^{1}$. Este curso se concibe para ser desarrollado de manera desescolarizada y en el tiempo del instructor, evitando así desmejorar las metas de los Centros de Formación y generando una cultura de aprendizaje permanente.

La meta inicial de este curso es la generación de una cultura informática en los instructores, que enriquezca su quehacer pedagógico y que permita la innovación permanente de los programas de formación del SENA y que lleve a la institución a ser competitiva en la sociedad del conocimiento.

Además del desarrollo de los multimedios, Ihardun se encargó de formar 60 tutores para impartir el curso, la formación se realizó con el material multimedial en un $70 \%$ de su desarrollo y con la retroalimentación de los tutores se logró contextualizar y enriquecer el material. La formación de dichos tutores se realizó en tres fases: la primera fue una semana presencial donde además de dar a conocer el material se trataron temas relacionados como los medios audiovi-suales y la desescolarización de adultos. En la fase dos se trabajó de manera desescolarizada apoyada espe- cialmente en correo electrónico y herramientas básicas, en una página web, entre las que se contaba con un tablero de anuncios y espacios para descarga de documentos de apoyo. Para la fase final se reunieron los tutores tres días durante los cuales se consolidaron experiencias, entre las cuales sobresalió el aislamiento que sentían los tutores por las continuas fallas de conexión a la red que impidieron contacto permanente con las personas en España.

Para iniciar el proceso de formación de todos los instructores y teniendo en cuenta que 60 tutores es un número muy pequeño para llevar a cabo de manera rápida a nivel de todo el país, se imparte desde la Dirección General - División de Investigación y Desarrollo Técnico Pedagógico -, el lineamiento a todas las Regionales y Seccionales de formar uno o dos tutores en cada uno de los Centros de Formación, esta actividad debe ser desarrollada por los 60 tutores iniciales, de acuerdo con el plan de multiplicación presentado. Esta formación adicional de tutores se realizó en el año 2000 , llegando a un número aproximado de 200 tutores en todo el país y se distribuyeron 1.000 copias del material multimedial para todos los Centros, para ser facilitado al instructor en calidad de préstamo o para ser duplicado por poseerse licencia ilimitada de reproducción del material.

A mediados del año 2000 se dio inicio definitivo a la formación de los instructores del SENA en ese curso y se da como lineamiento a todos los centros, para la planeación de actividades del

\footnotetext{
${ }^{1}$ El SENA cuenta con 25 Regionales y Seccionales en todo el país y con 114 Centros de Formación encargados de desarrollar los programas de formación tanto presénciales como desescolarizados.
} 
Plan de Mejoramiento Continuo de Instructores año 2001, el programar el curso inicialmente para todos sus instructores y posteriormente ofrecerlo al medio externo.

En esta primera etapa se identifica que, dado que los materiales multimediales no son la totalidad del curso y que es necesario tener una tutoría que permita obtener los resultados esperados, Internet es la tecnología que permite una interacción asincrónica necesaria para que los instructores puedan desarrollar el curso en su tiempo, por la diversidad de horarios que estos manejan y la posibilidad de actualizar y complementar los contenidos de los CD Roms a costos muy bajos de distribución.

La formación en TIC apoyada en ambientes virtuales de aprendizaje:

A nivel de la Dirección General del SENA y dando respuesta a la política de estado denominada Agenda de Conectividad - El S@Ito a Internet -, es aprobado en el Consejo Directivo Nacional el Programa de Teleinformática y Formación Virtual, en el cual se estructura el proyecto denominado Comunidad Educativa Virtual. Este proyecto tiene como objetivo Crear ambientes virtuales que permitan una nueva estrategia de formación profesional acorde con los requerimientos educativos de la Era de la Información, sin barreras de tiempo ni espacio, con nuevos modelos pedagógicos y didácticos que privilegien la autoformación, la investigación, la resolución de problemas y el trabajo colaborativo.

Este proyecto se inicia buscando unificar criterios sobre qué es la formación en ambientes virtuales de aprendizaje, establecer estándares nacionales para la construcción de este tipo de cursos y líneas claras para prescribir medios que la apoyen.

Mediante la integración de un grupo seleccionado entre los tutores destacados en el proceso inicial, que poseen un trayectoria en sus centros como líderes del manejo de informática educativa y muchos de ellos responsables del codesarrollo del curso de NTIC, se desarrolla un trabajo que inicia con una semana de trabajo presencial en la cual se toman avances realizados a nivel Regional y se estructuran los documentos:

- $\quad$ Estándares para Cursos en Ambientes Virtuales de Aprendizaje.

- Lineamientos Nacionales del SENA para la Formación Deses colarizada en Ambientes Virtuales de Aprendizaje.

- La Selección y el Diseño de Medios de Comunicación para el proceso de Enseñanza Aprendizaje.

Estos documentos se enviaron a las Divisiones de Investigación y Desarrollo Técnico Pedagógico de las Regionales y Seccionales para que se trabajaran con lo Centros de influencia y se envaran todas las observaciones y aportes correspondientes, aclarando que éstos podrán ser modificados periódicamente dado el avance vertiginoso de las tecnologías involucradas, del cambio de roles de alumnos y tutores para dar respuesta a los cambiantes requerimientos de formación que demanda el mundo empresarial para ser competitivo.

Este mismo grupo se encargó de poner en práctica estos documentos mediante la estructuración de una prueba piloto interna, para lo cual el curso de NTIC resulta ser el más adecuado, pues se cuenta con los materiales básicos de 
contenidos, con los tutores, que además ya conocen la herramienta Intralearn, y los instructores SENA a nivel nacional deben cumplir, dentro de sus objetivos laborales, con el desarrollo del curso, lo que hace que exista una motivación para realizarlo. Adicionalmente, se está en la etapa final de la construcción de una red nacional institucional, que unirá en una red WAN los 114 Centros de formación.

Es así como los 6 temas del curso son distribuidos para diseñar y desarrollar las guías que servirán al alumno para autogestionar el aprendizaje de los contenido multimediales de los CD Roms, la creación de espacios colaborativos, tanto síncronos como asíncronos, tales como el Email, el chat, los foros, los documentos de apoyo y enlaces de interés, que son fácilmente creados en el software (Intralearn).

Los temas del curso son:

- Tecnología y sociedad.

- Representación e ideología.

- Comunicación y educación.

- Medios audiovisuales.

- Redes e internet.

- Multimedia.

Las reglas básicas sobre las que se crearon los contenidos son:

- La duración de cada uno de los 6 temas o módulos de formación que componen el curso es de 4 semanas, en cada una de las cuales se dividirá el contenido del módulo.

- Se trabajarán 3 foros, el primero es el técnico en el cual se resolverán dudas de manejo del software y se darán "truquitos" para un mejor desempeño del equipo y de la conexión. Un segun- do foro servirá de espacio de socialización libre a los participantes del curso, es un espacio para compartir escritos, enlaces o cualquier tipo de información que no esté relacionada directamente con el contenido. El tercer foro es el temático, en el cual el tutor planteará un tema relevante al contenido que se está trabajando durante la semana y en donde el alumno deberá participar de manera permanente.

- Se seleccionarán las actividades más relevantes del curso, teniendo en cuenta que se espera que un alumno destine un mínimo de dos horas diarias para un total del 10 horas semanales y de 40 horas en total para el tema. Como es bien sabido, esta estimación de tiempo es muy relativa, ya que dependerá de los conocimientos previos del alumno, de la velocidad de asimilación de los contenidos y del interés de profundización de los temas tratados. De todas formas como es necesario tener un marco de tiempo para el manejo administrativo del curso, a los alumnos se les da un tiempo de 4 semanas en total para finalizar cada módulo.

- Se seguirá teniendo como base de contenidos los CD Roms, el grueso de la formación se realiza de manera offline, permitiendo al alumno hacer la formación a su ritmo, sin los altos costos de conexión a Internet y sólo hacerla para realizar las actividades de colaboración en el servidor ${ }^{1}$.

- Se buscará unificar la presentación gráfica de los 6 módulos, para que los alumnos no tengan inconvenientes al finalizar un módulo e iniciar el siguiente.

El desarrollo de las guías tomó un mes, en el cual se solicitó a los Jefes inmediatos de los tutores asignar por lo me- 
nos la mitad del tiempo de trabajo a esta actividad. Se seleccionaron parejas de tutores para esta labor, buscando las fortalezas de conocimientos de cada módulo y se mantuvo comunicación permanente entre los integrantes del grupo.

Una vez finalizado el mes de trabajo, se realizó una reunión de tres días para hacer una revisión final de este. En esta reunión se detectó que no se habían seguido los lineamientos estructurados y que prevalecía la visión de cada pareja de tutores en su tema. Por lo tanto se concedió una semana más para hacer los ajustes antes de iniciar la prueba piloto, pero esta unificación nunca se dio.

En paralelo con el desarrollo de las guías se realizó la convocatoria en los Centros de Formación donde laboraban los tutores, para que los instructores de las diferentes especialidades tecnológicas se inscribieran en los grupos que conformarían la prueba piloto.

\section{Estructuración de la prueba piloto:}

La prueba piloto está estructurada de la siguiente manera:

- Los dos tutores que desarrollaron cada módulo se encargan de tutorizar cada uno 20 alumnos en su tema durante 4 semanas, como son 6 módulos de formación en total serán 12 tutores, y como cada uno tutoriza 20 alumnos, en total se tendrán en formación 240 alumnos.

- Los alumnos inician con el módulo que maneja el tutor de su centro, ya que en el módulo inicial es muy importante conocer y dominar la interfaz del software de administración de contenidos (Intralearn).

- Todos los cursos iniciarán simultáneamente con una videoconferencia que permitirá a los alumnos conocer las reglas de juego globales y a los tutores a nivel nacional.

- Al final del curso, es decir en el sexto módulo, el alumno habrá tenido 6 tutores diferentes ubicados geográficamente en diferentes partes del país. Es importante anotar que el tutor del centro al que pertenece el alumno deberá servir de apoyo durante el desarrollo de los 6 módulos como tutor y dinamizador del proceso.

La prueba piloto se inició oficialmente en el mes de agosto del año 2001 y deberá finalizar en el mes de mayo de 2002. Este período de tiempo es mayor a los 6 meses que daría una simple operación matemática de número de módulos por su duración en semanas, dado que en el SENA existe la figura de vacaciones colectivas, lo que hace que los meses de diciembre y enero deban ser manejados de manera diferente.

\section{Conclusiones de lo observado en desarrollo de la prueba piloto}

Necesidad de un equipo coordinador central:

El coordinador de la prueba piloto a nivel nacional es el Grupo de Teleinformática y Formación Virtual de la Dirección General, que no cuenta con profesionales de dedicación exclusiva para la prue-

\footnotetext{
${ }^{1}$ La dirección del servidor en el cual se está realizado la prueba piloto es http://virtual.sena.edu.co
} 
ba, lo que ha causado inconvenientes en cuanto al control de unificación de contenidos, el seguimiento a los tutores y la sistematización total de la prueba.

Su gstión se ha centrado en labores administrativas de creación de cuentas de tutores, inscripción de alumnos a los cursos y apoyo técnico para solucionar problemas con el software. Además, de la responsabilidad por la operatividad técnica de la conectividad y de los servidores está en manos de la División de Organización y Sistemas de la Dirección General a nivel global y de las Divisiones de Sistemas de las Regionales a nivel local. Esta separación de responsabilidades ha generado impactos negativos en la prueba, pues dicha división hace programación de ajustes y/o cambios que ha generado hasta una (1) semana continua de interrupción en la prueba, o no presta con prontitud la asistencia técnica requerida a las interrupciones del servicio, lo que ha causado desmotivación y deserciones significativas.

Igualmente, la falta de equipos en los Centros de Formación para el trabajo docente genera traumatismo, aunque estos cuentan con un número considerable de PCs, estos están ubicados en aulas o en áreas administrativas, lo que hace casi imposible el acceso de los instructores a estos recursos. Por esto, dentro del Programa de Teleinformática se tiene el Proyecto de Aula Abierta de Informática, en el cual se busca que las aulas de informática de los centros estén disponibles a alumnos e instructores por lo menos 16 horas al día. De todas formas, es importante crear en los Jefes de Centro la necesidad de acercar cada vez más al instructor al uso de estas tecnologías, como apoyo a su quehacer pedagógico.

Lo antes expuesto, ha permitido concluir que aunque la tecnología no debe ser el fin del aprendizaje apoyado en medios informáticos y telemáticos, sí es fundamental que la tecnología opere de manera óptima para lograr los resultados esperados. En el caso de la formación en ambientes virtuales de aprendizaje se requiere de un servicio $7 \times 24$ $x 365$, con niveles de procesamiento adecuados, para lo cual es necesario contar con un grupo de profesionales expertos en teleinformática y una infraestructura robusta.

Igualmente, es necesario contar con un líder pedagógico que permita orientar de manera permanente la estructuración de contenidos, las evaluaciones y el papel del tutor.

\section{Dificultad en los cambios de roles:}

Definitivamente uno de los mayores inconvenientes detectados en la prueba tiene que ver con los roles, tanto de tutor como de alumno, pues se siguen repitiendo comportamientos de la formación presencial poco adecuados y muy arraigados culturalmente.

Podemos exponer los siguientes puntos:

Por parte del Tutor aún se observa:

- Formación centrada en el profesor y no en el alumno, esta actitud se vivencia en foros donde el tutor concluye y no deja generar una interacción adecuada, con el cierre de contenidos con fechas taxativas, con la falta de respuesta oportuna a los requerimientos del alumno.

- Formación memorística, con ejercicios y evaluaciones que no motivan al estudiante a investigar y a crear, sino a contestar sobre el contenido de los CDs. Es más, el mismo tutor no investiga, no enriquece cada vez más el módulo con nuevos documentos y sitios de interés.

- Falta de interés por conocer las nuevas herramientas y posibilidades 
que tiene a su alcance, ya que estando a punto de finalizar la prueba y luego de seis meses después de haber iniciado la prueba no se conoce bien el software con que se trabaja, ni se participa en las áreas colaborativas de la denominada "Sala de Tutores"

Por parte del Alumno:

- No se responsabiliza de su proceso de aprendizaje, continúa esperando las "órdenes" del tutor.

- Deserta fácilmente ante cualquier adversidad, lo que denota falta de motivación en el proceso.

- Tiene fuertes deficiencias en el manejo de la tecnología, lo que dificulta el proceso.

- No maneja adecuadamente la lectura y la escritura, que son fundamentales para utilizar de manera adecuada los ambientes colaborativos. Esto también se observa en algunos tutores.

Estas deficiencias se han venido tamizando con el transcurrir de la prueba y existen tutores líderes que han venido marcando la pauta. Es interesante notar cómo en la "sala de tutores" se han generado discusiones sobre la misma prueba, se han dado aportes importantes para solucionar inconvenientes, pero aún falta mucha cultura de uso de estos medios virtuales en los tutores, ya que de los 12 solo 5 son asiduos participantes de estos espacios.

Alta deserción:

Un resultado desafortunado de esta prueba es la alta deserción de alumnos, existen grupos en donde sólo se cuenta con 4 alumnos, por lo tanto ha sido necesario unificar grupos y realizar tutorías compartidas.

Los grupos que se han mantenido son aquellos en los que el tutor del Centro al que pertenecen ha permanecido de manera permanente como dinamizador y ha contado con el apoyo del Jefe del Centro. Esto demuestra que aún existe una necesidad del contacto cara a cara por parte de los alumnos, pues en muchos casos los apoyos a través de Internet son demasiado impersonales para nuestra cultura latina.

Aquellos grupos que no cuentan con esta dinamización han tenido grandes bajas, destacándose uno en el cual su tutor no pudo continuar con el proceso por problemas de salud, en donde sólo subsiste un alumno.

Muchos de los alumnos que han desertado de uno o dos módulos tendrán la posibilidad de finalizar su curso, pues una vez concluida la prueba piloto se hará una convocatoria y se realizarán estos módulos.

\section{Problemas administrativos:}

Dado que esta es una estrategia de formación nueva en el SENA, no existen procesos y procedimientos establecidos para:

- Determinar el número óptimo de alumnos por curso, aunque este deberá variar según la complejidad del tema. Para la prueba piloto, como ya se dijo, se conformaron grupos de 20 alumnos por tutor.

- El control de tiempo del docente, ya que este puede realizar su trabajo fuera del Centro de Formación, aquí es más importante trabajar por resultados que por horas. En la prueba piloto existen diversidad de situaciones, estas van desde la desprogramación total, de me- 
dio tiempo, del tiempo necesario para un curso presencial de la misma intensidad y en algunos casos el tutor ha asumido este como un trabajo adicional. Esta diversidad se debe a que aunque hay un lineamiento de desprogramar al docente dos horas diarias para tutoría, los Jefes de Centro son autónomos en el tema. Vale la pena resaltar que los tutores destacados no son aquellos que tienen más tiempo programado.

- No existen procesos aprobados para realizar inscripción y certificación en línea, por lo que en la prueba piloto ha sido necesario realizar los mismos procedimientos que para una formación presencial en cuanto a inscripciones, reporte de notas, etc.

\section{La respuesta a nivel nacional:}

La respuesta frente al uso de ambientes virtuales de aprendizaje ha sido muy positiva en el SENA, pues se han recibido múltiples solicitudes para iniciar más cursos de formación en NTIC al igual que para desarrollar cursos en otras áreas tecnológicas. Muchas de estas solicitudes se han pospuesto en espera de poder contar con datos que permitan un ajuste real a los lineamientos nacionales y en poder solucionar y consolidar la infraestructura informática y telemática del SENA, para no repetir los errores cometidos.

Dentro del proyecto de Comunidad Educativa Virtual se tiene previsto no solo consolidar el desarrollo de cursos en ambientes virtuales - aula virtual -, sino que esta se complementará con biblioteca, laboratorio, oficina y espacios de socialización libres.

\section{La evaluación de la prueba piloto:}

Para una evaluación final de esta prueba piloto en el mes de mayo de 2002, se está desarrollando un formato de evaluación general, tanto para alumnos como para tutores, cuyos resultados serán tabulados para mejorar el curso y para actualizar los lineamientos nacionales.

Adicionalmente, en paralelo, se han iniciado procesos de formación con docentes de instituciones externas, usando el mismo material y los lineamientos de la prueba piloto, es necesario contrastar los resultados arrojados por la evaluación de la prueba y la de estos cursos antes de sistematizar los resultados obtenidos, ya que muchos instructores no dan la importancia necesaria al curso por ser interno.

Los resultados de la prueba piloto deberán ser también contrastados con los resultados de los primeros cursos que se presenten al público en otras áreas diferentes a la pedagogía y en las cuales es crucial para el alumno adquirir las competencias ofrecidas como medio para garantizar un mejor nivel de vida, pues es bien sabido que los instructores y docentes en general son muy reticentes al cambio.

\section{REFERENCIAS BIBLIOGRÁFICAS}

BARRIGA E. , 2000, Aula Virtual, Bucaramanga - Colombia : Editorial Ltda.

GALVIS PANQUEVAA., 1998, RIE, Ambientes Virtuales para Participar en la Sociedad del Conocimiento, Volumen 11, No. 2, páginas 247-260.

GALVIS PANQUEVAA., 1998, RIE, Educación para el Siglo XXI Apoyada en Ambientes Educativos, Lúdicos, Creativos y Colaborativos, Volumen 11, No. 2, páginas 169-192.

OSORIO GÓMEZL.A. y SÁNCHEZA., 2000, RIE, Ambientes Colaborativos en Ludomatica, Volumen 13, No.1, páginas 41-57. 